\title{
Multisession CyberKnife Radiosurgery for the Treatment of Advanced-Stage Intracranial Extended Juvenile Nasopharyngeal Angiofibroma
}

\author{
Shinichiro Miyazaki ${ }^{1}$, Raphael Barrera ${ }^{2}$, Cordelia Sharma \\ 1. CyberKnife Center, Shinyurigaoka General Hospital 2. Department of Surgery, Long Island Jewish \\ Medical Center 3.
}

$\square$ Corresponding author: Shinichiro Miyazaki, miyazaki1953123@yahoo.co.jp Disclosures can be found in Additional Information at the end of the article

\section{Abstract}

Background: A case of a large advanced stage juvenile nasopharyngeal angiofibroma (JNA) treated with combined temporally and volume fractionated CyberKnife radiosurgery (CKRS) is presented. The optimal modality for treating advanced JNA continues to be controversial because of the complications associated with surgical resection and the potential delayed morbidity of radiotherapy (RT) in a pediatric age group.

Clinical Presentation: CKRS treatment was delivered with a volume fractionation method. First, for the lower two-thirds of the tumor occupying the nasal cavity, pterygopalatine fossa, maxillary sinus, and ethmoid sinus, CKRS was administered in five fractionated treatments of 7.0 Gy to the $71.7 \%$ prescription isodose. This lower part of the tumor volume was $56.4 \mathrm{~cm}^{3}$. Next, for the upper one-third of the tumor occupying the sphenoid sinus, infratemporal fossa and intracranial left cavernous sinus, CKRS was administered in five fractionated treatments of $6.2 \mathrm{~Gy}$ to the $72.1 \%$ prescription isodose. This upper part of the tumor volume was $25.2 \mathrm{~cm}^{3}$. Post-treatment, a gradual reduction in the whole tumor volume was observed continuously over the course of follow-up, and the patency of the internal carotid artery was restored. Radiation-related complications were limited to nasal mucosal crust formation.

Conclusion: A case of a large advanced stage JNA treated with CKRS using combined temporal and volume fractionated CKRS is presented. Follow-up revealed reasonable continuous reduction in tumor volume and the normal patency of the internal carotid artery without any radiation-related complication except for nasal mucosal crust formation. Longer follow-up is needed to evaluate late morbidity and long-term disease control.

Published 09/30/2011

\section{Copyright 2011}

Miyazaki et al. This is an open access article distributed under the terms of the Creative Commons Attribution License CC-BY 3.0., which permits unrestricted use, distribution, and reproduction in any medium, provided the original author and source are credited.
Categories: Neurosurgery

Keywords: cyberknife, hypofractionated radiotherapy, juvenile nasopharyngeal angiofibroma, radiosurgery, isodose

\section{Introduction}

JNA is a rare, histologically benign tumor of predominantly adolescent males. The most frequently presenting symptoms were nasal obstruction with recurrent epistaxis. The treatment modality in JNA depends on the extent of the disease. For uncomplicated tumors without intracranial involvement, surgical resection is the treatment of choice [1-3]. For large tumors 
with intracranial extension, the potential for surgical morbidity is very high because of their relation to major vasculature and cranial nerves at the base of the skull, and it may still leave residual disease needing postoperative RT $[1-2,4]$. The RT had been used as the primary modality of treatment for extensive lesions with good control rates with acceptable morbidity [1-2, 4-11]. We present a case of advanced staged huge JNA that was treated by multisession CKRS with a volume fractionated technique. The tumor demonstrated an excellent response to this radiosurgical technique as manifested by a gradual reduction in tumor volume on imaging. Treatment morbidity was minimal.

\section{Abbreviations}

JNA- Juvenile nasopharyngeal angiofibroma

CKRS- CyberKnife radiosurgery

RT- Radiotherapy

MRA- Magnetic resonance angiography

\section{Case Presentation}

A 23-year-old man presented with a six year history of worsening nasal obstruction, recurrent nasal bleeding, and left swelling of his cheek. He had a past history of severe, recurrent, spontaneous epistaxis, persistent nasal obstruction, and discharge. Embolization had been performed 10 times in the past six years, each time after the occurrence of vigorous nasal bleeding. The patient was evlauated at a local university hospital where the diagnosis of an advanced stage JNA was established. Given both the operative risk with such a massive tumor and its anatomic extension into the intracranial cavernous sinus as well as the pterygoparatine and infratemporal fossas, open surgical treatment was deferred. The patient was referred to our hospital for the consideration of radiosurgery. After detailed imaging evaluation on December 12, 2008 (Figure 1A, 1B), we elected to do multisession CKRS with volume fractionation technique as an alternative to surgical tumor resection. 


\section{Cureus}

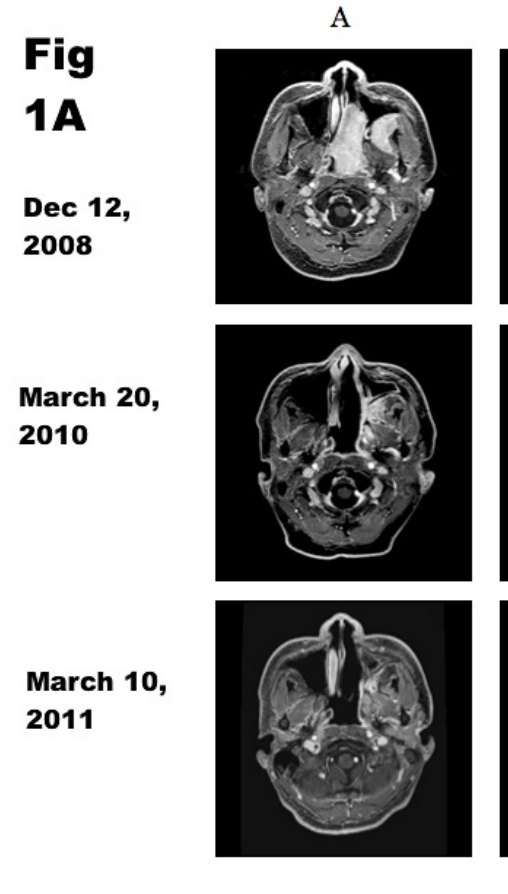

A

Fig

$1 B$

Dec 12,

2008

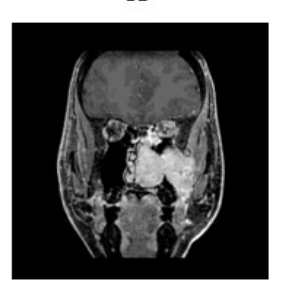

March 20, 2010
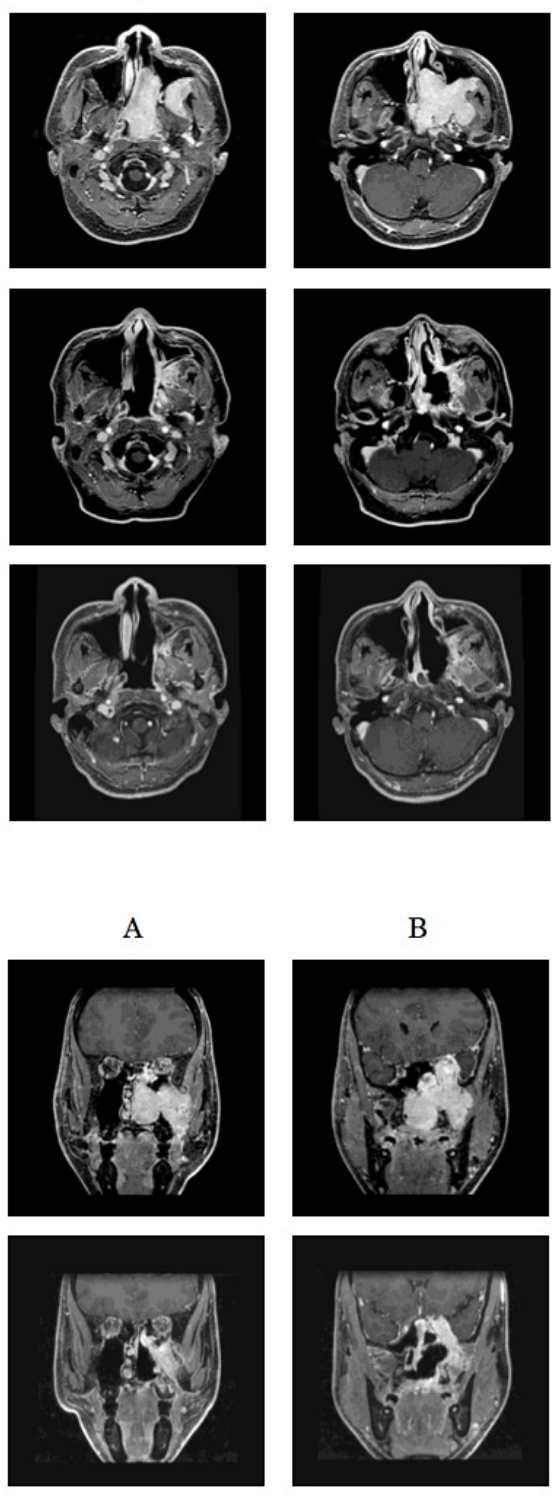

B
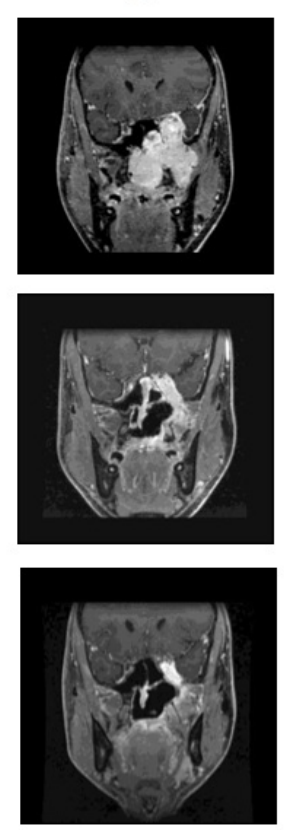

C
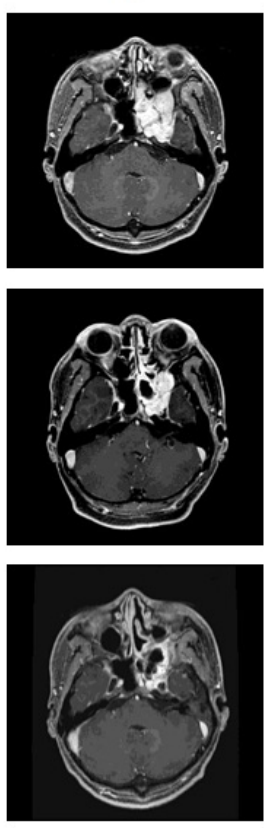

C
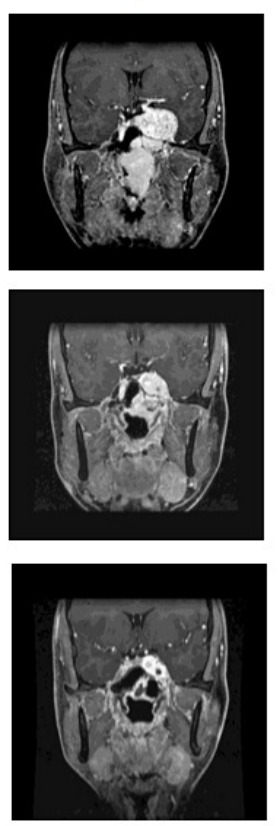

$\mathrm{D}$
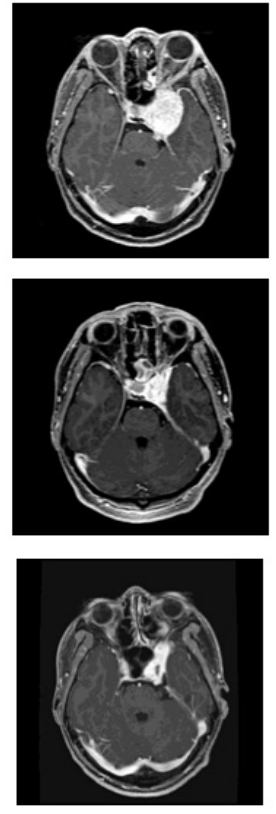

$\mathrm{D}$
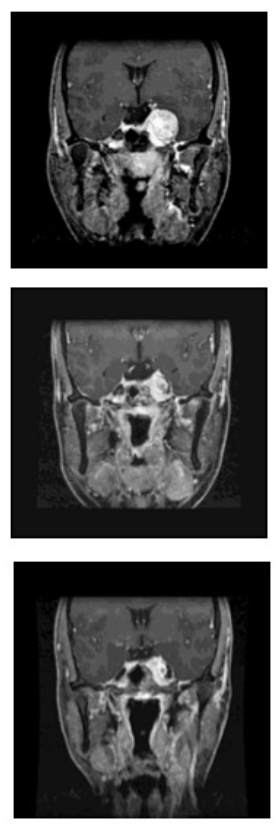

FIGURE 1: Gadolinium enhanced T1-weighted MR of axial (a), coronal (b) image, in the 23-year-old advanced stage juvenile nasopharyngeal angiofibroma (JNA).

These images delineated brightly enhancing soft tissue mass arising from the soft palate, left nasal cavity, nasopharynx with extension onto the infratemporal, palatine and left inframaxillary region. The mass was extending to the ethmoidal, sphenoidal sinuses eroding into the left cavernous sinus. The large JNA images observable and gradual volume reduction before (Dec.12, 2008) and after CKRS (Mar.20, 2010 and Mar. 10, 2011) . 


\section{Cureus}

\section{Intervention}

Because of huge tumor volume and advanced stage (Andrews staging IVb) (Table 1) with intracranial extension, multisession CKRS was done in addition to tumor volume fractionation technique. First, for the lower two-thirds of the tumor occupying the nasal cavity, pterygopalatine fossa, maxillary, and ethmoid sinus, CKRS was administered in five fractionated treatments of $7.1 \mathrm{~Gy}$ to the $71.7 \%$ prescription isodose (Figure $2 \mathrm{~A}$ ). The tumor volume was $56.6 \mathrm{~cm}^{3}$. Next, for the upper one-third of the tumor occupying the sphenoid sinus, infratemporal fossa, and intracranial left cavernous sinus, CKRS was administered in three fractionated treatments of 10.4 Gy to the $72.1 \%$ prescription isodose (Figure $2 b$ ). 


\section{Cureus}
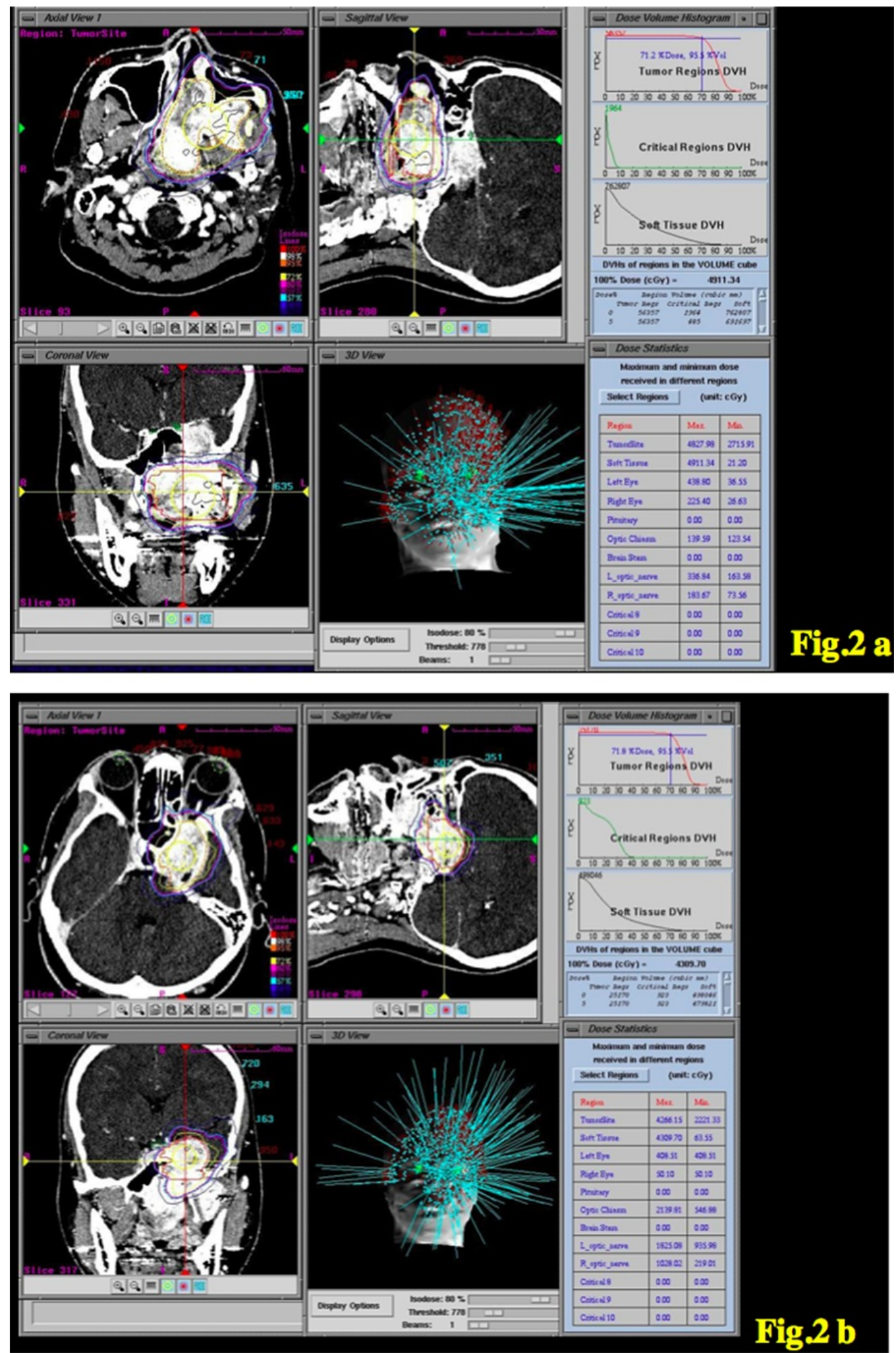

FIGURE 2: Advanced stage JNA CyberKnife treatment plan (a, b).

The whole tumor volume (too large to do CyberKnife radiosurgery (CKRS) in one time treatment. Then the tumor volume was divided into two parts and one was lower two-thirds $(a, 56.6 \mathrm{~cm} 3)$ and the other was upper one-third $(b, 25.2 \mathrm{~cm} 3)$. Axial, coronal and sagittal images are presented along with isodose line and critical structure contours. On the upper right, dosevolume histogram $(\mathrm{DVH})$ is shown. On the lower right, maximum and minimum dose to the 


\section{Cureus}

The tumor volume was $25.2 \mathrm{~cm}^{3}$. After all, the total tumor volume, including from down to nasal cavity and up to cavernous sinus region, was $81.8 \mathrm{~cm}^{3}$. After completed all treatment plans, close clinical and imaging follow-up was done in regular monthly intervals, and observable continuous reduction in tumor volume was demonstrated without radiation-related complication, except for nasal mucosal crust formation several months after the treatment. The last follow-up 27 months after the treatment on March 10, 2011 showed almost 95\% tumor volume decreased, including intracranial cavernous sinus portion (Figure 1A, 1B) and preserving normal cranial nerve function, normal pituitary endocrinological function, and normal patency of carotid arteries (Figure 3).

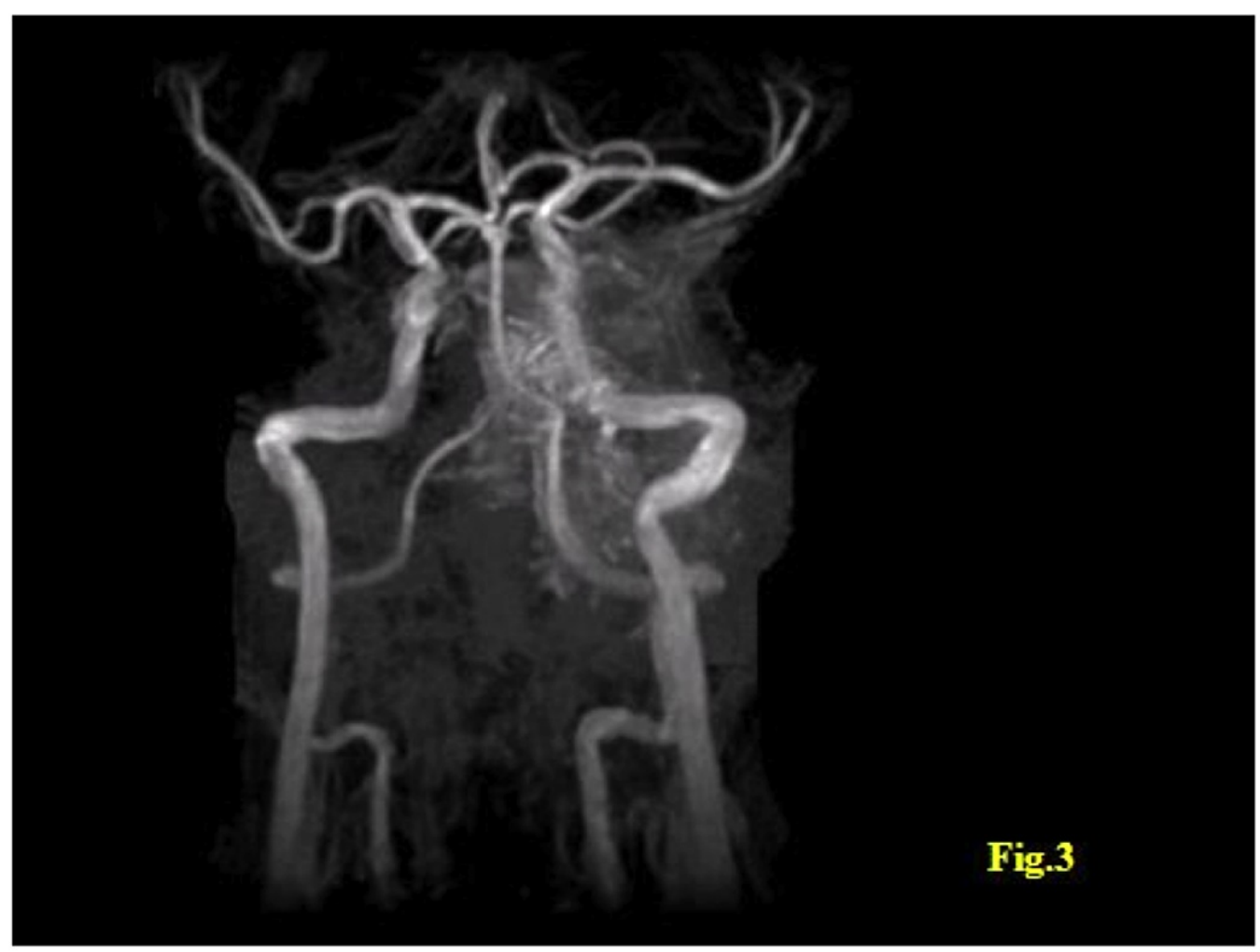

FIGURE 3: MRA obtained the last follow-up period on March 20, 2010 demonstrated the normal patency of internal carotid artery.

\section{Discussion}

JNA is a rare, highly vascular benign tumor of predominantly adolescent males. Although it is considered histologically benign, the tumor may exhibit locally aggressive and destructive behavior and eroding into adjacent structures, such as skull base and infiltration of the carotid arteries, cavernous sinus, and cranial nerves. As a consequence, significant morbidity and even death has occurred from intracranial extension and fatal epistaxis. The incidence of intracranial involvement has been reported to occur in six to over $90 \%$ of all the cases $[1,6]$. Tumors are classified according to their location and growth pattern with the staging [12]. This presenting 


\section{Cureus}

case was representing as Andrews staging IVb (Table 1).

Limited to the nasopharynx and nasal cavity, bone destruction negligible, or limited to the sphenopalatine foramen

II Invades the pterygopalatine fossa or the maxillary, ethmoid or sphenoid sinus with bone destruction

Illa Invades the infratemporal fossa or the orbital region without intracranial involvement

IIIb Invades the infratemporal fossa or orbit with intracranial extradural (parasellar) involvement

IVa Intracranial intradural tumour without infiltration of the cavernous sinus, pituitary fossa, or optic chiasm

IVb Intracranial intradural tumour with infiltration of the cavernous sinus, pituitary fossa, or optic chiasm

\section{TABLE 1: Andrews classification of JNA}

Andrew's (modified Fisch) classification

For cases with noninvasive lower stage group, it is agreed that evolved procedure of transnasal endoscopic resection can offer high rates of cure with minimal morbidity compared with more traditional open procedures [2]. For more advanced tumors with intracranial extension, as in our case, standard treatment options are open craniofacial surgery or definitive RT $[2-3,6]$.

The advanced lesions with intracranial extensions are a therapeutic challenge because of their close relationship with various critical structures, such as major vasculature, cranial nerves at the base of the skull, pituitary gland, optic apparatus, and hypothalamus, and their propensity for recurrence. There is a consensus in the literature that advanced disease with radiographic evidence of intracranial extension or those lesions seemed incompletely resectable without significant morbidity can be efficaciously treated with external-beam RT with acceptable morbidity [1-2, 4-11]. A significant concern related to RT is late toxicity, such as second malignancy [7], hypopituitalism [1, 7], cataract formation [1-2, 4, 7, 11], optic neuropathy or retinopathy, dental caries secondary to the xorostomia [8], and nasal dryness and crusting. Excellent local tumor control and considerably reduced morbidity as compared with the older radiotherapy series can be obtained in conformal radiotherapy series [5-6, 9]. Conformal radiotherapy can potentially reduce complications with significant dose reduction to the surrounding normal organs. It is an important consideration for JNA patients who are young and expected to live an active life without impairment caused by late radiation toxicity. Compared with conventionally fractionated radiotherapy, radiosurgical methods also have the advantage of sparing normal critical organ tissue as mentioned above. Multisession RS is an attractive, noninvasive treatment option for patients with large residual or recurrent or inoperable benign skull base tumors [13]. We prefer to use fractionated or multisession CKRS for extended tumor of the skull base as presented here. In the follow-up period, it is important to note that the tumor response to RT is often slow and that a persistent, stable residual abnormality should be considered controlled unless there is evidence of progression [2]. Although it seems safe and effective, more experience and longer follow-up periods are necessary to validate the optimal dose and fractionation scheme for the treatment of these complex shaped rare lesions.

\section{Conclusions}


A case of huge advanced stage JNA treated with fractionated CKRS, in addition to volume fractionation technique, is presented. The case showed reasonable continuous reduction in tumor volume with normal patency of internal carotid artery without any radiation-related complication, except for nasal mucosal crust formation. Determining optimal modality for advanced lesions continues to be a controversial problem because of complications associated with surgical resection and potential delayed morbidity of radiation therapy. This is the first reported case of advanced-stage JNA ( Andrews IVb) primarily treated with multisession CKRS radiosurgery. Longer follow-ups are needed to evaluate late morbidity and long-term disease control.

\section{Additional Information \\ Disclosures}

Human subjects: Consent was obtained by all participants in this study. Shioda Memoy Hospital issued approval. Conflicts of interest: In compliance with the ICMJE uniform disclosure form, all authors declare the following: Payment/services info: All authors have declared that no financial support was received from any organization for the submitted work. Financial relationships: All authors have declared that they have no financial relationships at present or within the previous three years with any organizations that might have an interest in the submitted work. Other relationships: All authors have declared that there are no other relationships or activities that could appear to have influenced the submitted work.

\section{References}

1. Lee JT, Chen P, Safa A, Juillard G, Calcaterra TC: The role of radiation in the treatment of advanced juvenile angiofibroma. Laryngoscope. 2002, 112:1213-1220.

2. McAfee WJ, Morris CG, Amdur RJ, Weming JW, Mendenhall WM: Definitive radiotherapy for juvenile nasopharyngeal angiofibroma. Am J Clin Oncol. 2006, 29:168-170.

3. Renkonen S, Hagstroem J, Vuola J, Niemelae M, Porras M, Kivivuori SM, Leivo I, Makitie AA: Changing surgical management of juvenile nasopharyngeal angiofibroma. Eur Arch Otorhinolaryngol. 2011, 268:599-607. 10.1007/s00405-010-1383-z

4. Reddy KA, Mendenhall WM, Amdur RJ, Stringer SP, Cassisi NJ: Long-term results of radiation therapy for juvenile nasopharyngeal angiofibroma. Am J Otolaryngol. 2001, 22:172-175.

5. Beriwal S, Eidelman A, Micaily B: Three-dimensional conformal radiotherapy for treatment of extensive juvenile angiofibroma: Report on two cases. ORL J Otorhinolaryngol Relat Spec. 2003, 65:238-241.

6. Chakraborty S, Ghoshal S, Patil VM, Oinam AS, Sharma SC: Conformal radiotherapy in the treatment of advanced juvenile nasopharyngeal angiofibroma with intracranial extension: An institutional experience. Int J Radiat Oncol Biol Phys. 2010, 80:1398-404.

7. Cummings BJ, Blend R, Keane T, Fitzpatrick P, Beale F, Clark R, Garrett P, Harwood A, Payne $\mathrm{D}$, Rider W: Primary radiation therapy for juvenile nasopharyngeal angiofibroma . Laryngoscope. 1984, 94:1599-1605.

8. Fields JN, Halverson KJ, Devineni VR, Simpson JR, Perez CA: Nasopharyngeal angiofibroma. Effiicacy of radiation therapy. Radiology. 1990, 176:263-265.

9. Kuppersmith RB, The BS, Donovan DT, Mai WY, Chiu JK, Woo SY, Bultler EB: The use of intensity modulated radiotherapy for the treatment of extensive and recurrent juvenile angiofibroma. Int J Pediatr Otorhinolaryngol . 2000, 52:261-268.

10. McGahan RA, Durrance FY, Parke RB, Easley JD, Chou JL: The treatment of advanced juvenile nasopharyngeal angiofibroma. Int J Radiat Oncol Biol phys. 1989, 17:1067-1072.

11. Robinson AC, Khoury GG, Ash DV, Daly BD: Evaluation of response following irradiation of juvenile angiofibroma. Br J Radiol. 1989, 62:245-247.

12. Andrews JC, Fisch U, Valavanis A, Aeppli U, Makek MS: The surgical management of extensive nasopharyngeal angiofibroma with infratemporal approach. Laryngoscope. 1989, 99:429-437.

13. Tuniz F, Soltys SG, Choi CY, Chang SD, Gibbs IC, Fischbein NJ, Adler JR Jr.: Multisession Cyberknife stereotactic radiosurgery of large, benign cranial base tumors: Preliminary study. Neurosurgery. 2009, 65:898-907. 10.1227/01.NEU.0000359316.34041.A8 